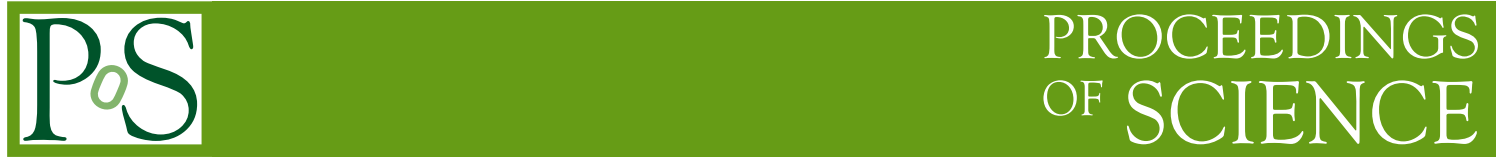

\title{
Radiative corrections to Quantum Hall Effect
}

\author{
Alexander Penin ${ }^{* \dagger}$ \\ Department of Physics, University of Alberta \\ E-mail: apenin@phys.ualberta.ca
}

We consider the quantum Hall effect in quantum electrodynamics and find a deviation from the quantum mechanical prediction for the Hall conductivity due to radiative antiscreening of electric charge in an external magnetic field

RADCOR 2009 - 9th International Symposium on Radiative Corrections (Applications of Quantum Field Theory to Phenomenology)

October 25-30 2009

Ascona, Switzerland

\footnotetext{
* Speaker.

${ }^{\dagger}$ This work is supported by the Alberta Ingenuity foundation and NSERC.
} 
The quantum Hall effect (QHE) [1,2] is a remarkable phenomenon remaining in the focus of experimental and theoretical research over the last three decades. The study of the QHE led to development of new fundamental physical concepts [3,4]. At the same time the QHE plays a crucial role in metrology and determination of fundamental constants [5].

In the QHE, in sharp contrast to the prediction of classical electrodynamics, the conductivity of the two-dimensional electron system in a strong transverse magnetic field at low temperature has plateaus as a function of the magnetic field strength. At these plateaus the conductivity is given by integer or specific fractional multiples of $R_{K}^{-1}$, where $R_{K}$ is a universal parameter known as the von Klitzing constant. A simple quantum mechanical consideration of the noninteracting electron gas relates it to the fine structure constant ${ }^{1}$

$$
R_{K}^{-1}=2 \alpha
$$

A remarkable property of a two dimensional electron system in magnetic field is that this naïve result is stable against all kinds of perturbations which do not result in a qualitative change of the Landau spectrum. This has been proven first in Ref. [6] (see also Ref. [7]) by an elegant use of gauge invariance. Later, a relation of the Hall conductivity to the topological invariants of the adiabatic ground state space has been established $[8,9,10]$. Much work has been done to find a possible deviation from Eq. (1) (see e.g. [11]). However, leaving aside finite temperature and edge effects, no universal corrections have been found and Eq. (1) is currently considered to be exact [5]. This would distinguish the quantum Hall conductance as one of a very few characteristics of many-particle interacting quantum systems exactly predicted by theory. On the other hand the exact relation (1) would allow for determination of the fine structure constant with a priori zero theoretical uncertainty.

However in quantum electrodynamics (QED) quantum field effects lead to a deviation from the quantum mechanical prediction for the Hall conductance [12]. The physics behind this phenomenon is in a modification of the local electromagnetic coupling of electrons due to vacuum polarization by highly virtual electron-positron pairs in a strong magnetic field, which can roughly be described as radiative antiscreening of the electric charge. Following Ref. [6] we consider the Hall current $I$ around an asymptotically large loop of a two-dimensional ribbon subject to a timeindependent locally homogeneous magnetic field $B$ and an electric field $E$. The spatial vectors $\vec{I}$, $\vec{B}$, and $\vec{E}$ are orthogonal to each other and the magnetic field is normal to the ribbon surface, see Fig. 1. For the futher analysis it is convenient to introduce an auxiliary magnetic flux $\Phi$ through the loop. The Hall conductivity $R_{H}^{-1}$ is defined by the equation $I=R_{H}^{-1} V$ where $V$ is the potential drop across the ribbon. In QHE it is given by $R_{H}^{-1}=v R_{K}^{-1}$ where the filling factor $v$ can be either integer [1] or fractional [2]. We focus on the integer QHE since the case of fractional $v$ can be understood as the integer QHE for fractionally charged quasiparticles [3]. In QED the dynamics of an electron in a magnetic field $B$ can be described by the effective Hamiltonian of the following form

$$
\mathscr{H}=e^{*} A_{0}-\frac{\vec{D}^{2}}{2 m^{*}}+\delta \mathscr{H},
$$

\footnotetext{
${ }^{1}$ Throughout the paper, if it is not explicitly stated otherwise, we adopt the system of units used in particle physics, where $\hbar=c=1$ and $\alpha=e^{2} /(4 \pi)$.
} 


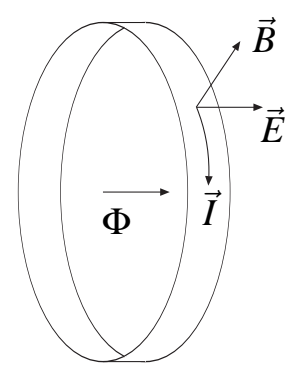

Figure 1: Geometry of the Hall current. The size of the loop is much larger than any other scale of the problem and the magnetic field is homogeneous near the surface of the ribbon.

where $A_{0}$ is the potential of the electric field $E, \vec{D}$ is the spatial covariant derivative, $e^{*}\left(m^{*}\right)$ stands for the effective charge (mass) of the electron, and $\delta \mathscr{H}$ represents the radiative and relativistic corrections as well as the interaction with the medium. The parameters of the Hamiltonian can be systematically computed in QED as a series in $\alpha$ and $\beta$ within the effective theory approach [13]. The quantum Hall conductivity is known to be independent of $m^{*}$ and $\delta \mathscr{H}[6,8]$. Thus the only source of the corrections to Eq. (1) is electron coupling to the external fields. This coupling is modified by vacuum polarization through creation of hard virtual electron-positron pairs. In the absence of a magnetic field this effect is reabsorbed by the on-shell renormalization of the physical electron charge $e$. For a nonvanishing magnetic field the vacuum polarization cannot be "renormalized out" and the effective charge does differ from $e$. Since the magnetic field $B$ explicitly breaks down the Lorentz invariance, the effective charges are in general different for different external fields. For the calculation of the Hall conductivity we need beside $e^{*}$ another effective charge $e^{\prime}$, which parametrizes the coupling of the electrons to the vector potential of the auxiliary magnetic flux in the covariant derivative $\vec{D}=\vec{\partial}-i e^{\prime} \vec{A}^{\Phi}+\ldots$

The effective charges are determined by the behavior of the vacuum polarization tensor $\Pi_{\mu v}(q)$ at small four-momentum transfer $q$. By using the integral representation of Refs. $[14,15]$ it is straightforward to derive the leading variation of the polarization tensor due to the magnetic field in the limit $q \rightarrow 0$, which reads

$$
\begin{aligned}
& \delta \Pi_{\mu v}(q)=-\frac{\alpha}{\pi} \beta^{2} \frac{1}{45}\left[2\left(g_{\mu \nu} q^{2}-q_{\mu} q_{v}\right)\right. \\
& \left.-7\left(g_{\mu \nu} q^{2}-q_{\mu} q_{v}\right)_{\|}+4\left(g_{\mu \nu} q^{2}-q_{\mu} q_{v}\right)_{\perp}\right] .
\end{aligned}
$$

The correction to the polarization tensor is transverse because of the gauge invariance. At the same time the Lorentz invariance is broken and Eq. (3) includes the transverse projectors in the "parallel" $\left(q_{0}, \vec{q}_{\|}\right)$and "orthogonal" $\left(\vec{q}_{\perp}\right)$ two-dimensional subspaces of the whole four-dimensional Minkowskian momentum space $\left(q_{0}, \vec{q}\right)$. Here $\vec{q}_{\|}$and $\vec{q}_{\perp}$ components correspond to the spatial momentum parallel and orthogonal to the magnetic field, respectively. The polarization tensor determines both the correction to the local coupling of electrons to the electromagnetic potential and the correction to the photon propagator, which is non-trivial since the external magnetic field changes the photon dispersion law [15]. 


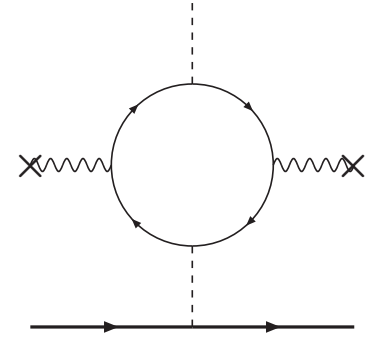

(a)

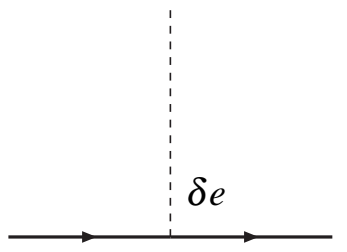

(b)

Figure 2: Feynman diagrams in full QED $(a)$ and in the nonrelativistic effective theory $(b)$ representing the antiscreening of the electric charge in the external magnetic field. The arrow lines correspond to the free electron propagators. The bold arrow lines correspond to the electron propagating in the external magnetic field. The dashed lines represent the electric potential, the crossed wavy lines represent the external magnetic field, and $\delta e=e^{*}-e$.

Let us now consider the effective charge $e^{*}$, which parametrizes the interaction of the electron to the homogeneous electric field. The first two terms in square brackets of Eq. (3) result in a modification of the static Coulomb potential between two pointlike charges [16]

$$
V(\vec{r})=\frac{\alpha}{r}\left[1+\frac{\alpha}{\pi} \beta^{2}\left(\frac{2}{45}-\frac{7}{90} \sin ^{2} \theta\right)\right],
$$

where $\theta$ is the angle between $\vec{B}$ and $\vec{r}$, i.e. the Coulomb interaction in the presence of the magnetic field becomes anisotropic. Taking an infinite uniformly charged plane as a source of $E$ and using the potential (3) for the electron interaction with the charge density, one gets the following result

$$
e^{*}=e\left[1+\frac{1}{45} \frac{\alpha}{\pi} \beta^{2}\right]
$$

where the only nonvanishing contribution is due to the first Lorentz covariant term of Eq. (3). The angular dependent term in Eq. (4) represents the correction to the Coulomb photon propagator and its contribution to Eq. (5) vanishes. Thus the vacuum polarization in the magnetic field enhances the electron coupling to the electric field which generates the Hall current. Graphically the effect is represented by the Feynman diagrams in Fig. 2.

Similar effect occurs in the case of the effective charge $e^{\prime}$. The vector potential of the auxiliary magnetic flux has only $\vec{A}_{\perp}$ component and its momentum has only $\vec{q}_{\|}$component. Thus only the first term of Eq. (3) contributes to the corresponding coupling and one gets $e^{\prime}=e\left[1+\alpha \beta^{2} /(45 \pi)\right]$, i.e. $e^{\prime}=e^{*}$. Note that $e^{\prime}$ is exactly the parameter which appears in the quantization condition for the auxiliary magnetic flux through the contour of the Hall current. Hence in the presence of the magnetic field $B$ the "effective" flux quantum becomes $\Phi_{0}^{\prime}=2 \pi / e^{\prime}$ or

$$
\left(\Phi_{0}^{\prime}\right)^{-1}=\frac{e}{2 \pi}\left[1+\frac{1}{45} \frac{\alpha}{\pi} \beta^{2}\right]
$$

Now we are in a position to derive the correction to the quantum mechanical result for the Hall conductivity. In general, the Hall current is given by the integral of the current density over the 
ribbon cross section

$$
I=\int\left[j_{I}(\vec{r})+\delta j_{I}(\vec{r})\right] \mathrm{d} r_{E} \mathrm{~d} r_{B},
$$

where $\vec{r}=\left(r_{I}, r_{E}, r_{B}\right)$ is a vector with the components parallel to the $\vec{I}, \vec{E}$, and $\vec{B}$, respectively. A single electron contribution to the unperturbed current density can be written as follows

$$
j_{I}(\vec{r})=-i \frac{e}{m} \phi^{*}(\vec{r}) D_{I} \phi(\vec{r}),
$$

where $\phi(\vec{r})$ is the eigenfunction of the Hamiltonian (2). In QED the perturbation to the current density is due to the vacuum polarization. The Hall current flows in the orthogonal subspace and the corresponding correction to the vacuum polarization is given by the first and the last terms of Eq. (3). However, the last term of Eq. (3) does not vanish for $q^{2}=0$ and represent the change of the photon dispersion law rather than the correction to the current density, which is completely determined by the first Lorentz covariant term

$$
\delta j_{I}(\vec{r})=\frac{1}{45} \frac{\alpha}{\pi} \beta^{2} j_{I}(\vec{r}),
$$

The expression for the Hall current takes the following form

$$
I=\left(1+\frac{1}{45} \frac{\alpha}{\pi} \beta^{2}\right) \int j_{I}(\vec{r}) \mathrm{d} r_{E} \mathrm{~d} r_{B}
$$

The integral in Eq. (10) can be expressed through the derivative of the electron energy $\mathscr{E}$ in $\Phi$

$$
\int j_{I}(\vec{r}) \mathrm{d} r_{E} \mathrm{~d} r_{B}=-\frac{e}{e^{\prime}} \frac{\mathrm{d} \mathscr{E}}{\mathrm{d} \Phi}
$$

see e.g. Ref. [11]. Thus our final expression for the Hall current reads

$$
I=-\frac{\mathrm{d} \mathscr{E} t}{\mathrm{~d} \Phi}
$$

where $\mathscr{E}_{t}$ is the total energy of the electrons contributing to the current. As has been shown in Ref. [6], the flux $\Phi$ acts as a quantum pump: changing it by $n$ quanta $\Phi_{0}^{\prime}$ results in a net transfer of $n v$ electrons across the ribbon, which corresponds to an energy variation of $n v e^{*} V$. Thus for the Hall conductivity one gets

$$
R_{H}^{-1}=v \frac{e^{*}}{\Phi_{0}^{\prime}} .
$$

Putting together Eqs. $(5,6,13)$ we obtain the final expression for the von Klitzing constant

$$
R_{K}^{-1}=2 \alpha\left[1+\frac{2}{45} \frac{\alpha}{\pi} \beta^{2}\right]
$$

or in physical units

$$
R_{K}^{-1}=\frac{e^{2}}{2 \pi \hbar}\left[1+\frac{2}{45} \frac{\alpha}{\pi}\left(\frac{\hbar e B}{c^{2} m^{2}}\right)^{2}\right] .
$$

We would like to emphasize that the characteristic distance of the vacuum fluctuations resulting in the correction to the Hall conductivity is given by the electron Compton wavelength of order 
$10^{-12} \mathrm{~m}$, which is far smaller than the actual thickness of the layer where the electrons are localized, of order $10^{-8} \mathrm{~m}$. Thus the correction to $R_{K}$ is due to an intrinsically three-dimensional effect, which is not prohibited by the topological and gauge invariance arguments developed in two dimensions.

The correction term in Eq. (15) can be rewritten as follows

$$
\frac{2}{45} \frac{\alpha}{\pi}\left(\frac{B}{B_{0}}\right)^{2}
$$

where $B_{0}=c^{2} m^{2} /(\hbar e) \approx 4.41 \cdot 10^{9} \mathrm{~T}$. A typical value of the magnetic field in current experiments corresponds to $B / B_{0} \sim 10^{-8}$. Thus numerically Eq. (16) amounts to a tiny $10^{-20}$ correction.

\section{References}

[1] K. von Klitzing, G. Dorda, and M. Pepper, Phys. Rev. Lett. 45, 494 (1980).

[2] D.C. Tsui, H.L. Störmer, and A.C. Gossard, Phys. Rev. Lett. 48, 1559 (1982)

[3] R.B. Laughlin, Phys. Rev. Lett. 50, 1395 (1983).

[4] B.I. Halperin, Phys. Rev. Lett. 52, 1583 (1983).

[5] P.J. Mohr and B.N. Taylor, Rev. Mod. Phys. 77, 1 (2005), and references therein.

[6] R.B. Laughlin, Phys. Rev. B 23, 5632 (1981).

[7] B.I. Halperin, Phys. Rev. B 25, 2185 (1982).

[8] D.J. Thouless, M. Kohmoto, M.P. Nightingale, and M. den Nijs, Phys. Rev. Lett. 49, 405 (1982).

[9] B. Simon, Phys. Rev. Lett. 51, 2167 (1983).

[10] J.E. Avron and R. Seiler, Phys. Rev. Lett. 54, 259 (1985).

[11] D.R. Yennie, Rev. Mod. Phys. 59, 781 (1987).

[12] A. Penin, Phys. Rev. B 79, 113303 (2009), Erratum ibid. in press.

[13] W.E. Caswell and G.P. Lepage, Phys. Lett. B 167, 437 (1986).

[14] J. Schwinger, Phys. Rev. 82, 664 (1951).

[15] S.L. Adler, Ann. Phys. (N.Y.) 67, 599 (1971).

[16] Yu.M. Loskutov and V.V. Skobelev., Phys. Lett. A 36, 405 (1971). 